\title{
Behaviors of Yahisuli's Parents in Early Childhood Immunization in 2018
}

\author{
Ependja Towaka Antoine ${ }^{1}$, Baelo Foyo Vif ${ }^{1}$, Baombolia Lokangu \\ Zenon $^{1}$
}

${ }^{1}$ Higher Institute of Medical Techniques of Yangambi, DR Congo

*Corresponding author: Ependja Towaka Antoine: aependja@gmail.com

\section{OPEN ACCESS}

Citation: Ependija T.A., Baelo F.V., Baombolia L.Z. (2021) Behaviors of Yahisuli's Parents in Early Childhood Immunization in 2018.

Open Science Journal 6(2)

Received: $2^{\text {nd }}$ November 2020

Accepted: $27^{\text {th }}$ November 2020

Published: $2^{\text {th }}$ May 2021

Copyright: (C) 2021 This is an open access article under the terms of the Creative Commons Attribution License, which permits unrestricted use, distribution, and reproduction in any medium, provided the original author and source are credited.

Funding: The author(s) received no specific funding for this work

Competing Interests: The author has declared that no competing interests exists.

\section{Abstract:}

This descriptive cross-sectional study aims to determine the behavior of Yahisuli's parents in early childhood immunization in 2018. To collect data, we used a questionnaire administered to a non-probability convenience sample of 96 Yahisuli parents caring for a child aged 0 to 11 months. Descriptive analysis allowed us to compare our results with previous work. We recorded an average age of 32.8 years and a male/female sex ratio of 1.3 . More than $95 \%$ of the parents attended school, of which $58.5 \%$ had a high school education. The birth rank of the last child is between 3 and 5 with a sex ratio of 1.3 in favor of males. Nearly $15 \%$ of children have not been fully vaccinated. In this work, 55 out of 96 parents bring their children to a health facility to be vaccinated to ensure their protection $(76.4 \%)$ and prevention against infectious diseases (47.3\%). On the other hand, $42 \%$ do not adhere to vaccination because of ignorance, fear due to children's incessant crying after vaccination, adverse post-immunization events. When it came to vaccines, several misunderstandings were raised. These corroborate rumors observed under other skies. Thus, a descriptive and analytical study on the epidemiological surveillance of adverse effects of vaccines is indispensable in the province of Tshopo.

Keywords: Behavior, Vaccine, Vaccination, Childhood, Tshopo. 


\section{Introduction}

In the past, vaccines have elicited reactions from the population, linked to the political context of their administration, or to characteristics that are at odds with traditions, sometimes also to intrinsic manufacturing defects.

The changing natural history of infectious diseases and the resurgence of diseases that have almost disappeared make vaccination a central issue in general medicine. Numerous quantitative and qualitative studies have identified determinants of the parents' vaccination decision without providing an in-depth understanding of the motivation for the decision [1].

In the tropics, strengthened by the success of vaccines in reducing infectious diseases worldwide [2], public health organizations would like to complete the elimination of major cosmopolitan diseases (tuberculosis, poliomyelitis, measles), as well as to extend the benefits of vaccination to diseases specific to the geographical area, including parasitosis embedded in particular biotopes $[1,3]$.

However, apart from the challenges to be met by biomedicine, the expectations and vision of the populations concerned must also be taken into consideration. This is not only a legitimate effort, from a human rights perspective, to improve information and foster citizen autonomy, along a path that is developing before our eyes.

Fifty years after the end of colonization, certain reactions of populations in tropical areas reflect a crisis of confidence in vaccination programs driven from above and from outside. The consolidation and even improvement of the results achieved with vaccines and the extension of vaccination coverage to new diseases require a historical, sociological and anthropological reflection on the implementation of programs.

In several countries, the vaccine had entered into competition with an old method using smallpox directly to obtain immunity. In general, the proponents of the vaccine then opposed the modern vaccine par excellence to the archaic, random and dangerous variolization. In Algeria, doctors perceived that resistance to vaccinia was mainly rooted in opposition to the foreign invader. In India, for a long time the English did not hurry to upset the traditional variolization sponsored by the Brahmins. Hindus were resistant to collecting vaccine lymph from the sides of the sacred cow, and shunned operations conducted by vaccinators recruited from the lower castes [1].

The Indian example of variolization, carefully analyzed by historians, highlights that vaccination is never a neutral and anonymous routine operation, immune from suspicion in the community, except perhaps in the case of epidemic-related panic. The rest of the time, the population asks itself questions that are only apparently simple: who vaccinates whom, when, how and why?

Answering these questions is crucial to avoid vaccine resistance, or in modern terms, to predict its acceptability [3]. Even poorly educated populations are quite capable of making relevant clinical observations [4].

Today, one is never completely immune to a break in the cold chain, the spread of altered or defective products, and the sporadic occurrence of measles or polio in vaccinated children.

In DR Congo, despite progress in reducing under-five mortality from 158 deaths per 1,000 live births in 2007 to 104 in 2013, the situation remains worrisome in terms of the global situation and the achievement of sustainable development goals by 2030. This excess mortality is largely due to vaccinepreventable causes. Indeed, the estimated vaccination coverage in 2013 for all 
antigens was low: only $41 \%$ of children aged 12 to 23 months were fully vaccinated [5].

In the province of Tshopo, we observed cases of resistance during mass campaigns, particularly in the health zone of Yalimbongo, Opienge, Bafwasende and Yaleko. In these areas, communities such as the Kitawalists, Idomites, Catholic bongola motema, Nzambé Lumumba are very hostile to vaccination. In fact, during the 2017 supplementary immunization activities, OPV, a live attenuated vaccine, was administered to a child under five years of age in the Yaleko health zone, resulting in acute flaccid paralysis.

Although the health zone of Yahisuli is not spared from cases of resistance and/or refusal of vaccination based on certain suspicions when it comes to vaccines, we have observed that no study on the parents' behavior towards vaccination has been carried out.

It is in this context that we initiated this study to determine the behavior of Yahisuli's parents in the early childhood vaccination in 2018. Specifically, we wanted to describe the characteristics of the parents surveyed, identify the reasons for adherence and dropping out of vaccination as well as their judgments about misunderstandings around the vaccine.

\section{Methodology}

This descriptive cross-sectional study took place in the Yahisuli health zone in the province of Tshopo. This zone is mainly occupied by the Topoke, Lokele and Mbole tribes. Our population is composed of parents who are in charge of a child aged 0 to 11 months. Due to the lack of a reliable sampling list, we used a nonprobability convenience sampling of 96 subjects, using a self-administered questionnaire for data collection. Descriptive analysis based on headcount tables, percentage calculations and arithmetic mean allowed us to compare our results with previous work. Parental consent, confidentiality and anonymity were respected.

\section{Presentation of results}

\section{Characteristics of surveyed parents}

Age

Table I : Age distribution of parents surveyed at Yahisuli in 2018

\begin{tabular}{lcc}
\hline Age & Staff & $\%$ \\
\hline $52-58$ & 9 & 9,4 \\
$45-51$ & 7 & 7,3 \\
$38-44$ & 16 & 16,7 \\
$31-37$ & 17 & 17,7 \\
$24-30$ & 21 & 21,8 \\
$17-23$ & 26 & 27,1 \\
\hline Total & 96 & 100 \\
\hline
\end{tabular}


Parents aged 17 to 23 followed by those aged 24 to 30 are dominant. The average age is 32.8 years.

Gender

Table II: Gender distribution of parents surveyed at Yahisuli in 2018

\begin{tabular}{lcc}
\hline Gender & Staff & $\%$ \\
\hline Man & 54 & 56,3 \\
Woman & 42 & 43,7 \\
\hline Total & 96 & 100 \\
\hline
\end{tabular}

Sex ratio male $/$ female $=1.3$.

Level of education

Table III: Distribution of parents surveyed at Yahisuli in 2018 by Education Level

\begin{tabular}{lcc}
\hline Level of education & Staff & $\%$ \\
\hline Primary & 17 & 18,1 \\
Secondary & 55 & 58,5 \\
Superior & 22 & 23,4 \\
\hline Total & 94 & 100 \\
\hline
\end{tabular}

$58.5 \%$ of the subjects have a secondary school education compared to $23.4 \%$ with a higher education level.

Marital status

Table IV: Distribution of surveyed parents in Yahisuli in 2018 by marital status

\begin{tabular}{lcc}
\hline Marital status & Staff & $\%$ \\
\hline Married & 90 & 93,7 \\
Single & 6 & 6,3 \\
\hline Total & 96 & 100 \\
\hline
\end{tabular}

Our respondents are represented at $93.7 \%$ of the bride and groom.

Activities carried out

Table V: Distribution by occupation of parents surveyed at Yahisuli in 2018

\begin{tabular}{lcc}
\hline Occupation & Staff & $\%$ \\
\hline Cultivator & 20 & 20,8 \\
Housewife & 29 & 30,2 \\
Débrouillard & 7 & 7,3 \\
State agent & 40 & 41,7 \\
\hline Total & 96 & 100 \\
\hline
\end{tabular}

The majority of our respondents were government employees (41.7\%), followed by housewives $(30.2 \%)$ and farmers (20.8\%). 
Religion

Table VI: Distribution of parents surveyed in Yahisuli in 2018 by religion

\begin{tabular}{lcc}
\hline Religion & Staff & $\%$ \\
\hline Christian & 89 & 92,7 \\
Kimbanguist & 6 & 6,3 \\
Kitawala & 1 & 1,0 \\
\hline Total & 96 & 100 \\
\hline
\end{tabular}

More than $90 \%$ of our respondents belong to Christianity.

Household size

Table VII: Distribution by household size of parents surveyed in Yahisuli in 2018

\begin{tabular}{lcc}
\hline Household size & Staff & $\%$ \\
\hline $14-16$ & 14 & 14,5 \\
$11-13$ & 9 & 9,4 \\
$8-10$ & 30 & 31,3 \\
$5-7$ & 26 & 27,1 \\
$2-4$ & 17 & 17,7 \\
\hline Total & 96 & 100 \\
\hline
\end{tabular}

The household size of the majority of our respondents is between 8 and 10 people $(31.3 \%)$ or an average of 8 people per household.

Gender of the last child in the family

Table VIII: Distribution of parents surveyed in Yahisuli in 2018 by the sex of their last child born alive

\begin{tabular}{lcc}
\hline Sex of last child & Staff & $\%$ \\
\hline Male & 55 & 57,3 \\
Female & 41 & 42,7 \\
\hline Total & 96 & 100 \\
\hline
\end{tabular}

The last child born living in $57.3 \%$ of the households surveyed was male. The male/female sex ratio was 1.3 .

Child's birth rank

Table IX: Distribution of surveyed parents in Yahisuli in 2018 by birth rank of last child

\begin{tabular}{lcc}
\hline Rank of birth of last child & Staff & $\%$ \\
\hline $15-17$ & 3 & 3,1 \\
$12-14$ & 3 & 3,1 \\
$9-11$ & 4 & 4,2 \\
$6-8$ & 26 & 27,1 \\
$3-5$ & 38 & 39,6 \\
$0-2$ & 22 & 22,9 \\
\hline Total & 96 & 100 \\
\hline
\end{tabular}


The birth rank of the last child of the parents surveyed ranged from 3 to 5 $(39.6 \%)$. The average birth rank is 5 .

Geographic accessibility

Table X: Distribution of Subjects by distance to travel to a health facility in Yahisuli in 2018

\begin{tabular}{lcc}
\hline Distance to go in Km & Staff & $\%$ \\
\hline$\geq 5$ & 16 & 16,6 \\
$2-4$ & 28 & 29,2 \\
$\leq 1$ & 52 & 54,2 \\
\hline Total & 96 & 100 \\
\hline
\end{tabular}

More than $50 \%$ of the parents surveyed are within one kilometer of a health facility.

\section{Parents' behaviour towards vaccination}

Vaccination adherence

Table XI: Opinion of Yahisuli's parents in 2018 on reasons for adhering to vaccination $(\mathrm{n}=57)$

\begin{tabular}{lcc}
\hline Reason for adherence to vaccination & Staff & $\%$ \\
\hline Prevention & 26 & 47,3 \\
Protection & 42 & 76,4 \\
Don't know & 6 & 10,9 \\
\hline
\end{tabular}

Protection (76.4\%) and prevention (47.3\%) are the main reasons for adherence to vaccination according to the parents surveyed.

Reason for dropping out of vaccination

Table XII: Opinion of Yahisuli's parents in 2018 on reasons for dropping out of vaccination $(\mathrm{n}=41)$

\begin{tabular}{lcc}
\hline Reason for dropping out & Staff & $\%$ \\
\hline The disease & 24 & 58,5 \\
Ignorance & 5 & 12,2 \\
Don't know & 17 & 41,5 \\
\hline
\end{tabular}

More than $55 \%$ of parents surveyed do not adhere to vaccination because of the effects observed in children after vaccination. 
Fully immunized children

Table XIII: Distribution of Yahisuli's parents in 2018 according to the number of fully immunized children

\begin{tabular}{lcc}
\hline Fully vaccinated children & Staff & $\%$ \\
\hline $15-17$ & 5 & 5,2 \\
$12-14$ & 2 & 2,1 \\
$9-11$ & 3 & 3,1 \\
$6-8$ & 12 & 12,5 \\
$3-5$ & 28 & 29,2 \\
$1-2$ & 31 & 32,3 \\
0 & 15 & 15,6 \\
\hline Total & 96 & 100 \\
\hline
\end{tabular}

$15.6 \%$ of the children of the parents surveyed had not been fully vaccinated. The average number of children fully vaccinated is 4 .

Anxiety about vaccination

Table XIV: Reasons given by Yahisuli's parents in 2018 for fear of vaccines $(n=96)$

\begin{tabular}{lcc}
\hline Reasons for fear of vaccines & Staff & $\%$ \\
\hline Pleurs & 45 & 46,9 \\
Side effects & 41 & 42,7 \\
Misunderstandings & 5 & 5,2 \\
\hline
\end{tabular}

Our respondents' fears about bringing their children to immunization reside mainly in the incessant crying after vaccine administration (46.9) and adverse post-immunization events $(42.7 \%)$.

Method of vaccine administration

Reason of preference for oral administration

Table XV: Parents' opinion on reasons for preference of oral vaccine administration $(\mathrm{n}=70)$

\begin{tabular}{lcc}
\hline Reasons for oral administration of vaccines & Staff & $\%$ \\
\hline No side effects & 45 & 64,3 \\
Easy to administer & 22 & 31,4 \\
Don't know & 3 & 4,3 \\
\hline Total & 70 & 100 \\
\hline
\end{tabular}

The majority of parents prefer the oral route because it does not cause side effects $(64.3 \%)$. Ease of administration was also preferred by $31.4 \%$ of the parents surveyed. 
Reason for preference of administration by injection

Table XVI: Reasons given by parents for their preference for vaccine administration by injection $(n=26)$

\begin{tabular}{|c|c|c|}
\hline Reasons for injectable vaccine administration & Staff & $\%$ \\
\hline Effective & 8 & 30,8 \\
\hline Immediate effects & 18 & 69,2 \\
\hline Total & 26 & 100 \\
\hline $\begin{array}{l}\begin{array}{l}\text { Immediate effects } \\
\text { preference. }\end{array} \\
\text { Category of vaccinators }\end{array}$ & main & for in \\
\hline Preferred vaccinators & Staff & $\%$ \\
\hline Nurse & 78 & 81,3 \\
\hline Doctor & 11 & 11,5 \\
\hline Midwifery/midwife & 7 & 7,2 \\
\hline Total & 96 & 100 \\
\hline
\end{tabular}

Nurses are preferred vaccinators by $81.3 \%$ of the parents surveyed.

Misunderstandings about vaccines

Table XVIII: Opinion of parents surveyed on misunderstandings about the round of vaccinations or immunization $(\mathrm{n}=96)$

\begin{tabular}{lcc}
\hline Misunderstanding about vaccines or vaccination & Staff & $\%$ \\
\hline Toxic products & 53 & 55,2 \\
Vaccines hide dark intentions & 43 & 44,8 \\
Products that have nothing to do with the disease & 28 & 29,2 \\
Sterilize the population & 23 & 23,9 \\
Waste of silver for polio & 23 & 23,9 \\
Operation contrary to divine providence & 18 & 18,8 \\
Means to enrich the health authorities & 18 & 18,8 \\
\hline
\end{tabular}

$55.2 \%$ of parents think that vaccines are toxic. The population suspects that vaccines provided free of charge by large organizations are hiding dark intentions $(44.8 \%)$. The others think that they are products that have nothing to do with the disease $(29.2 \%)$, sterilize the population $(23.9 \%)$, etc. 
Respondents' suggestions

Table XIX: Suggestions from Yahisuli's parents to health managers and their partners $(\mathrm{n}=96)$

\begin{tabular}{lcc}
\hline Parents' wishes & Staff & $\%$ \\
\hline Support in drugs and equipment & 59 & 61,5 \\
Staff motivation & 14 & 14,5 \\
Take into account other basic needs & 9 & 9,4 \\
Raise community awareness about vaccines & 5 & 5,2 \\
No idea & 9 & 9,4 \\
\hline Total & 96 & 100 \\
\hline
\end{tabular}

More than $60 \%$ of the parents suggested that health managers and their partners provide support in terms of medicines and equipment for children.

\section{Discussion}

\section{Characteristics of parents}

Certain characteristics of the parents in charge of the children may explain immunization behaviors. They may also be responsible for not keeping immunization appointments and thus influence the follow-up of the child's immunization program. For example, age, education, marital status, religion, occupation, number of children under five, media exposure, area of residence, decision-making power, etc., may also influence the child's immunization schedule.

In their study of the individual and environmental factors associated with full immunization of children in rural Burkina Faso, Sia, Kobiane, Sondo and Fournier [18] found a link between the occupations of mothers and their spouses and the full immunization of their children.

On the subject of education level, it should be noted that this reflects the progress made by a population in terms of schooling and even development. It contributes to the improvement of the living conditions of household members and society in general. The level of education also influences reproductive behavior, the use of modern contraception, health behavior, and hygiene and nutrition habits.

Data from DHS-DRC II showed that 19 percent of Congolese women have never attended school and therefore have no education, compared to 8 percent of men. Thus, the proportion of uneducated women is about twice as high as that of men [5].

A 2006 study conducted in Benin in 2006 on factors associated with parental behavior in immunization found that $74.2 \%$ of parents were between 21 and 35 years of age, of which $50.8 \%$ were in school and less than $20 \%$ were unemployed. Birth rank was second with extremes ranging from 1 to 8 [6]. 
Ouedraogo L.T. et al. 19] also found that maternal illiteracy (73.3\%) influenced adherence to childhood immunization. Low education levels can be a handicap to access to information on immunization. The higher level of education of fathers could be an important asset if they are involved in sensitization activities.

The proportion of married people observed in this work is consistent with the results of the DHS-DRC II survey. The survey found that more than six out of ten $(64 \%)$ lived in a union: $46 \%$ of married women and $18 \%$ in a consensual union [5].

Most of our respondents belong to Christianity. This is due to their numbers in the population. However, there is reason to suggest that, according to a study carried out in Benin in 2006, parents practicing the traditional religion (48.1\%), large households $(29.9 \%)$ and those whose child was the third born of the family (28.4\%) were not vaccinated [6].

Compared to the sex of the last child born alive, Makoutode et al. observed $50.2 \%$ of male children [6]. This corroborates the results of our work.

However, Ependja in a similar survey conducted in Opala found a predominantly female sex ratio. This is due to culture. Indeed, according to Bantu culture, a boy is the heir of the family, especially if he is the first born. Therefore, parents have a moral obligation to protect him against certain dubious practices that can negatively influence his fertility. References to rumors or misinformation about vaccines and immunization. This gives female children a chance to be seen in large numbers at the Expanded Programme on Immunization [7].

With respect to media exposure, it should be clarified that the household does not need to own a radio, television or buy a newspaper to have access to it. Many people may be able to listen to the radio or watch television at the home of friends or neighbors. These data are particularly important for the development of education, awareness and information dissemination programs in all areas, including health and, in particular, family planning [5]. The results of the 20132014 Demographic and Health Survey found that in DR Congo, men are usually more exposed to the media than women [5].

In this study, the proportion of parents who slept elsewhere or were absent from their homes is justified by the instability of households seeking survival for their families. The population is forced to travel long distances to follow the rotating market program. In addition, with the phenomenon of African solidarity in the event of death, some people are sometimes called upon to spend entire nights away from home. This does not exclude prayer vigils in churches.

The health facilities at Yahisuli are located less than a kilometer from the parents surveyed. However, previous studies have shown that parents do not adhere to vaccination when the health facility is more than a 15-minute walk away. This means that geographical accessibility, household size and religion are factors associated with parents' behavior in vaccinating their children. Accessibility and quality of care are factors that explain the therapeutic 
management of patients. Various studies have shown that, in general, health center utilization rates decrease as distances to be traveled increase [7].

Other studies carried out in various contexts have also shown that public health facilities are more solicited by populations that are close to them; and that the distances to be covered are relatively short. For example, in Nigeria, the distance is less than two kilometers, and in Ghana $70 \%$ of users are less than three thousand kilometers away [7].

\section{Parents' behavior towards immunization}

In this work, 55 out of 96 parents bring their children to a health structure to be vaccinated in order to ensure their protection and prevention against infectious diseases. On the other hand, 41 subjects do not adhere to vaccination because of disease and ignorance as well as fear due to children's incessant crying after vaccination, adverse post-immunization events.

Protection and prevention were also cited as the main reasons for adherence. Those who did not fully vaccinate their children cited fatigue, field work, religion, paralysis, and the ineffectiveness and inconvenience of vaccines. These factors led to $15.6 \%$ of children not being fully vaccinated.

These results corroborate those found by Ependja in the rural health zone of Opala. About $68 \%$ of parents do not bring their children to a health facility to be vaccinated for fear of anal lesions $(32.8 \%)$ and diseases (18.8\%). The low level of knowledge of the subjects about vaccination in Opala was associated with nonadherence as it found $31.2 \%$ of non-respondents and $76.5 \%$ of children fully vaccinated. These factors constitute in our opinion a high risk of non-adherence to the immunization program. Ignorance is therefore a factor not to be neglected in the fight against behaviors unfavorable to vaccination [7].

Fear of anal injury, disease, crying and side effects are not dismissed. Indeed, recently, the province of Tshopo is confronted with the resurgence of malaria and anal lesions co-infection. This can be observed in children from 0 to 59 months of age by anal redness relieved by chloramphenicol mixed with alkaloids from tomato leaves in local application. For the other health zones, this phenomenon is new and causes deaths among children under 5 years of age. Unlike the Opala health zone where the disease has been known since time immemorial; allusion made to the song dedicated to this pathology. For the parents, the appearance of this abnormal phenomenon defying modern medicine would be due to vaccination $[20]$.

The disease referred to in the responses of our respondents is only the fever observed in children when certain live attenuated vaccines were administered, notably Bacille de Calmette and Guérin, measles vaccine and DTPHepBHib. These vaccines are given by injection and can induce a mild rise in body temperature. This does not rule out the fear of paralysis of a limb [7].

Regarding vaccination status, Magatte et al. 21] in Senegal, in a comprehensive cross-sectional survey of immunization dropouts among 562 
mothers of children aged 10-23 months, found that the proportion of fully immunized children was statistically related to mothers' knowledge of the immunization schedule and the sex of the child. They also found that male children were more likely to be fully immunized. Jennifer [22] found a relationship between birth rank and child immunization status.

Regarding vaccinators and the route of vaccine administration, Moulin said that each vaccine is unique in its presentation, its mode of administration (by injection or by mouth). Injection is preferred in some cultures while the oral route is, depending on the case, appreciated for its ease and analogy with food or depreciated because of its banality. Scarification can be as easily accepted or suspected as a disturbing marking of the body. The personality of the vaccinator by social status, ethnicity, gender, etc. may also be involved [1].

\section{Misunderstandings about vaccines}

When it came to vaccines or vaccination, several misunderstandings were raised by our respondents. These corroborate the rumors observed under other skies. A good example of a misunderstanding is the turmoil triggered by the neonatal tetanus vaccination campaign in eastern Cameroon after the WHO decision to eliminate the disease in 1989. This vaccination had been openly offered to young women in reproductive age. This break with the unisex nature of vaccinations raised suspicions in a region already in turmoil: were they not vaccines aimed at sterilizing the population? Misinformation turned to tragedy; children fled from school, a murder took place, a riot atmosphere reigned for a few days, young girls became pregnant just to check their fertility [1].

1] The same rumor has in fact spread several times to other countries in Africa and Asia about vaccines unrelated to tetanus. Rumors about vaccines that are sterilizing, toxic or simply inactive have one thing in common: distrust of the major powers that are suspected of limiting the demographic vitality of the people under the guise of immunization aid [8].

8] Yemen and the Philippines have witnessed sporadic violence, following miscarriages that occurred in the population after vaccination campaigns.

In 2003, in Nigeria, a highly unstable country in the midst of the WHOsponsored campaign to eradicate childhood paralysis, polio immunization was denounced by the Sharia Supreme Council on a set of arguments against submission to divine providence and malicious interventions by Western powers [9].

Beyond paranoid assertions and rumors of plots, it is true that there are irregularities in the conduct of vaccinations: the interventions planned by the health authorities do not take into account the constraints of the population and the rhythms of work in the fields.

In Mossi country, infantile paralysis is a bird disease related to a jealous deity that should not be thwarted. 
The population asks itself the question: why devote so much money and energy to the prevention of a single disease, polio, which has few victims and only sporadically rages, while so many basic needs, such as drinking water or schools, remain unsatisfied?

For Chippaux, the behavior of populations is paradoxical only in appearance, when at the same time they suspect that the vaccines provided free of charge by large international organizations are hiding dark intentions and complain that they do not have access to the latest products of the pharmaceutical industry $[10]$.

10] Inspired by the vision of vaccines as "global public goods" distinct from ordinary medicines, charities have begun to support the spread of expensive new vaccines in tropical countries. 11] However, even this principled and laudable initiative may have appeared to the public as a decision taken over their heads, mobilizing funds and people that could have been better spent in other areas [12].

\section{Conclusion}

All in all, the vaccine tools are not exclusively at the service of the State or communities, but are first and foremost at the service of individuals who want to be protected and who wish to benefit from effective immunization corresponding to local risks. This is a historic undertaking that has its letters of glory, but which has also had its tragedies and blunders.

This paradox of vaccinology in the tropics is not inevitable. Thus, we propose to initiate a descriptive and analytical study on the epidemiological surveillance of the adverse effects of vaccines in the expanded program of routine vaccines or in mass campaigns.

\section{References:}

1.Moulin AM. Vaccination programs in tropical environments: Contribution of a social science reflection. Méd. Trop. 2007; 67: 335 - 339

2. Duclos P, Okwo-Bele JM. Global vaccine recommendations and policies: the role of WHO. Med Sci. 2007; 4: 409-416.

3.Levy-Bruhl D \& Cook J. Methodological approaches to the study of vaccine acceptability: example of three surveys conducted in West Africa. Health Social Sciences. 1993

4.Moulin AM. Vaccines: Social and Political Implications. Méd Mal Inf. 2003

5.Ministère du Plan et Suivi de la Mise en oeuvre de la Révolution de la Modernité (MPSMRM), Ministère de la Santé Publique (MSP) and ICF International, 2014. Demographic and Health Survey in the Democratic Republic of Congo 2013-2014. Rockville, Maryland, USA: MPSMRM, MSP and ICF International.

6.Makoutode M \& Mohamed S. Factors associated with parental behavior in early childhood immunization in Benin in 2006. Tropical Medicine 2007; 87(4):383.

7.Ependja A. Contribution to the study of factors associated with parental behavior in the vaccination of their children in Opala in 2011. Ann. Afr. Med. 2013 June ;6(2)

8.Cléments J \& Drake C. Combating anti-vaccination rumous; lessons learned from case studies in East Africa, case study report, Nairobi UNICEF Eastern and Southern Africa Regional Offic; 2002.

9. Yahya M. Polio vaccines "No thank You" Barriers to polio eradication in Northern Nigeria. Africa Affairs 2007; 106: 185-204. 
10.Chippaux JP. Epidemics in the South. Vaccine in the North. Science in the South. $2001 ; 11: 1$.

11. Moulin AM. Vaccines as Global Public Goods Occupational Heaith and Public Health Lessons from the Past. In MC Nelson-Challengs for the future. Stockholm: Arbetslivinstitutet ed; 2006

12.Muraskin W. Revolution in international Public Health? The origins and development of the Bill and Melinda Gates children's vaccine Program and the Global Alliance for Vaccines and immunization. Rochester: University Press Rochester; 2007

13.Parent of Châtelet I, Antona D, Waku-Kouomou D, Freymuth F, Maine C, Levy-Bruhl D. Measles in France in 2008: assessment of the compulsory declaration. Weekly Epidemiological Bulletin $2009 ; 39-40: 415-9$.

14.Schwarzinger M, Flicoteaux R, Cortaredona S, Obadia Y, Moatti JP. Determinants of individual acceptance of pandemic A(H1N1) 2009 vaccination in the French adult population. Weekly epidemiological bulletin 2010; 24-25-26:267- 71 .

15. Cotter S., Ryan F., Hegarty H, McCabe T. J., Keane E. Immunization: opinion of parents and health professionals in Ireland. Euro surveillance. 2003 June; 8(6): 145-150.

16.Anderson R. and May R. Infectious Diseases of humans: Dynamics and control. London: Oxford University Press; 1991

17.Geoffard P.-Y., Philipson T. "Disease eradication: Private versus public vaccination". American Economic Review. 1997 : 222-230.

18.Sia D., Kobiane J.F., Sondo B. \& Fournier P. Les facteurs individuels et du milieu de vie associés à la vaccination complète des enfants en milieu rural au Burkina Faso: une approche multi niveau. Cahiers Santé 2007; 17(4): 203-206.

19. Ouedraogo L.T. Determinant of non-compliance with the immunization schedule at the health district level: the case of the Boussé health district, Burkina Faso. Medicine and Infectious Diseases 2006; 36 :138-143

20.Ependja A. Coinfection malaria and anal lesions in children under five years of age: A new and uninvestigated phenomenon in the province of Tshopo. Sarrebruck: Editions Universitaires Européennes; 2018

21. Magatte N. \& coll. Factors in the drop-out of vaccination of children aged 10-23 months in Ndoulo, Senegal. Cahiers Santé. January-February-March $2009 ; 19(1)$

22.Jennifer N. Bondy. Identifying the determinants of childhood immunization in the Philippines. Vaccine 2009; 27 :169-175 